\title{
Letter to the Editor: Tele-rehabilitation of Back Pain Patients in Coronavirus Disease 2019 Pandemic
}

\author{
Hina Vaish \\ Maharishi Markandeshwar Institute of Physiotherapy and Rehabilitation, Maharishi Markandeshwar (Deemed to be University), Mullana, India
}

I have read the review "Creating backup management resources for spine care during the coronavirus disease 2019 pandemic" with great interest and appreciate the information provided [1]. I agree with most of what has been written in the operative and non operative cases section of the article. I agree to the report of increase in number of referral cases of back pain due to increase in the sedentary lifestyle, combined with the abnormal posture, increased household duties, raised workload and to the emphasis laid on telemedicine and long-distance consultations as the biggest contribution to healthcare in the current time. While agreeing with what has been detailed, I wish to highlight what the literature search brings forth about the utility of telerehabilitation specifically in non operative back pain patients.

Telerehabilitation refers to the use of telecommunication to provide distant support, assessment and rehabilitative interventions to the patients. Tele-rehabilitation has been found to be effective and has being practiced even before the Coronavirus disease 2019 times. However, the need of present times emphasizes to adopt tele-rehabilitation during the lockdown.

Evidence reports that home-based exercise therapy, when performed has been found to be effective for improving level of pain, functional capacity, and quality of life in patients with non-specific chronic low back pain [2]. Rehabilitation services by use of digital technology is cost effective, helps to maintain continuity of care, educate patients through remote consultation, also allows for performing a physical assessment, plan a targeted therapeutic exercise program, and monitor patients' progress, providing them continuous supervision and feedback.

Tele-rehabilitation has been used earlier in studies on back pain patients. Evidence from a case series reports that tele-rehabilitation and remote patient monitoring may be helpful in improving long-term management of patients with chronic low back pain [3]. Use of mobile-app platform of the McKenzie extension protocol has comparable clinical outcomes with the traditional clinic-based McKenzie therapy, and thus an effective supplementary platform for care of patients with low-back pain [4]. Thus, preliminary evidence suggests adopting tele-rehabilitation in substitute of face-to-face physical rehabilitation for improving pain scores and quality of life in patients affected by back pain [2-4].

However, literature also documents that home-based tele-rehabilitation managements with minimal supervision is the least preferred method as patients prefer less frequent physician consultation offered mainly through video communication, provided that they were provided

\footnotetext{
Received Jul 11, 2020; Accepted Jul 20, 2020

Corresponding author: Hina Vaish

Maharishi Markandeshwar Institute of Physiotherapy and Rehabilitation, Maharishi Markandeshwar (Deemed to be University), Mullana, Ambala, Haryana, 133207, India

Tel: +91-9450124758, E-mail: hinavaish@mmumullana.org; hina22vaish@gmail.com
} 
feedback and monitoring and face-to-face consultation [5]. Hence, virtual rehabilitation strategies should focus on individualized management. To maintain contact between patient and health care provider interactive apps with audio and video capabilities can be used. Video calls for monitoring of the patient, psychological counseling, explaining physical exercise, and even supporting patients with chronic back pain conditions to adhere to the plans.

Though, tele-rehabilitation is emerging as the first option of patient for rehabilitation services at home under professional supervision but level of education of patients, basic internet and instrument knowledge, internet connectivity and internet facilities in rural areas could be important challenges to be over come specially in developing and low resource countries.

\section{Conflict of Interest}

No potential conflict of interest relevant to this article was reported.

\section{ORCID}

Hina Vaish: https://orcid.org/0000-0001-6045-845X

\section{References}

1. Yadav SK, Kar BK, Banta A, Kumar A. Creating backup management resources for spine care during the coronavirus disease 2019 pandemic. Asian Spine J 2020;14:382-4.

2. Kanas M, Faria RS, Salles LG, et al. Home-based exercise therapy for treating non-specific chronic low back pain. Rev Assoc Med Bras (1992) 2018;64:82431.

3. Peterson S. Telerehabilitation booster sessions and remote patient monitoring in the management of chronic low back pain: a case series. Physiother Theory Pract 2018;34:393-402.

4. Mbada CE, Olaoye MI, Dada OO, et al. Comparative efficacy of clinic-based and telerehabilitation application of Mckenzie therapy in chronic low-back pain. Int J Telerehabil 2019;11:41-58.

5. Cranen K, Groothuis-Oudshoorn CG, VollenbroekHutten MM, IJzerman MJ. Toward patient-centered telerehabilitation design: understanding chronic pain patients' preferences for web-based exercise telerehabilitation using a discrete choice experiment. J Med Internet Res 2017;19:e26. 